\title{
Avanços Científicos e Tecnológicos em Bioprocessos
}

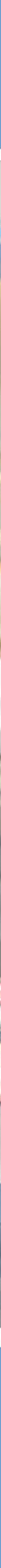


Alberdan Silva Santos

(Organizador)

\section{Avanços Científicos e Tecnológicos em Bioprocessos}

Atena Editora

2018 


\section{8 by Atena Editora \\ Copyright (C) da Atena Editora \\ Editora Chefe: Prof $^{\mathrm{a}} \mathrm{Dr}^{\mathrm{a}}$ Antonella Carvalho de Oliveira \\ Diagramação e Edição de Arte: Geraldo Alves e Natália Sandrini \\ Revisão: Os autores}

\section{Conselho Editorial}

Prof. Dr. Alan Mario Zuffo - Universidade Federal de Mato Grosso do Sul

Prof. Dr. Álvaro Augusto de Borba Barreto - Universidade Federal de Pelotas

Prof. Dr. Antonio Carlos Frasson - Universidade Tecnológica Federal do Paraná

Prof. Dr. Antonio Isidro-Filho - Universidade de Brasília

Prof $^{\mathrm{a}} \mathrm{Dr}^{\mathrm{a}}$ Cristina Gaio - Universidade de Lisboa

Prof. Dr. Constantino Ribeiro de Oliveira Junior - Universidade Estadual de Ponta Grossa

Prof $^{a}$ Dr $^{a}$ Daiane Garabeli Trojan - Universidade Norte do Paraná

Prof $^{a}$ Dr $^{\mathrm{a}}$ Deusilene Souza Vieira Dall'Acqua - Universidade Federal de Rondônia

Prof. Dr. Eloi Rufato Junior - Universidade Tecnológica Federal do Paraná

Prof. Dr. Fábio Steiner - Universidade Estadual de Mato Grosso do Sul

Prof. Dr. Gianfábio Pimentel Franco - Universidade Federal de Santa Maria

Prof. Dr. Gilmei Fleck - Universidade Estadual do Oeste do Paraná

Prof $^{a} \operatorname{Dr}^{\mathrm{a}}$ Girlene Santos de Souza - Universidade Federal do Recôncavo da Bahia

Prof $^{a} \mathrm{Dr}^{\mathrm{a}}$ Ivone Goulart Lopes - Istituto Internazionele delle Figlie de Maria Ausiliatrice

Prof. Dr. Julio Candido de Meirelles Junior - Universidade Federal Fluminense

Prof. Dr. Jorge González Aguilera - Universidade Federal de Mato Grosso do Sul

Prof $^{a}$ Dr $^{a}$ Lina Maria Gonçalves - Universidade Federal do Tocantins

Prof $^{a}$ Dr $^{\mathrm{a}}$ Natiéli Piovesan - Instituto Federal do Rio Grande do Norte

Prof $^{a} \mathrm{Dr}^{\mathrm{a}}$ Paola Andressa Scortegagna - Universidade Estadual de Ponta Grossa

Prof $^{a}$ Dr $^{\mathrm{a}}$ Raissa Rachel Salustriano da Silva Matos - Universidade Federal do Maranhão

Prof. Dr. Ronilson Freitas de Souza - Universidade do Estado do Pará

Prof. Dr. Takeshy Tachizawa - Faculdade de Campo Limpo Paulista

Prof. Dr. Urandi João Rodrigues Junior - Universidade Federal do Oeste do Pará

Prof. Dr. Valdemar Antonio Paffaro Junior - Universidade Federal de Alfenas

Prof $^{a} \mathrm{Dr}^{\mathrm{a}}$ Vanessa Bordin Viera - Universidade Federal de Campina Grande

Prof. Dr. Willian Douglas Guilherme - Universidade Federal do Tocantins

\begin{tabular}{|c|c|}
\hline & $\begin{array}{c}\text { Dados Internacionais de Catalogação na Publicação (CIP) } \\
\text { (eDOC BRASIL, Belo Horizonte/MG) }\end{array}$ \\
\hline \multirow[t]{10}{*}{ A946 } & $\begin{array}{l}\text { Avanços científicos e tecnológicos em bioprocessos [recurso } \\
\text { eletrônico] / Organizador Alberdan Silva Santos. - Ponta Grossa } \\
\text { (PR): Atena Editora, 2018. }\end{array}$ \\
\hline & Formato: PDF \\
\hline & Requisitos de sistema: Adobe Acrobat Reader \\
\hline & Modo de acesso: World Wide Web \\
\hline & Inclui bibliografia \\
\hline & ISBN 978-85-85107-47-5 \\
\hline & DOI 10.22533/at.ed. 475180110 \\
\hline & 1. Bioprocessos. 2. Bioquímica. 3. Biotecnologia. I. Santos, \\
\hline & $\begin{array}{ll}\text { Alberdan Silva. } & \text { CDD } 553.7\end{array}$ \\
\hline & Elaborado por Maurício Amormino Júnior - CRB6/2422 \\
\hline
\end{tabular}

O conteúdo do livro e seus dados em sua forma, correção e confiabilidade são de responsabilidade exclusiva dos autores.

2018

Permitido o download da obra e o compartilhamento desde que sejam atribuídos créditos aos autores, mas sem a possibilidade de alterá-la de nenhuma forma ou utilizá-la para fins comerciais. 